\title{
COVERINGS OF A TOPOLOGICAL SPACE
}

\author{
BY \\ R. S. PIERCE(1)
}

Introduction. One of the most interesting applications of the theory of lattices is point set topology. Beginning with the work of Stone [6], the close connection between the topological structure of a space $S$ and the algebraic structure of the lattice of open (or closed) sets of $S$ has been carefully investigated. A standard procedure in these investigations has been to represent the points of a space as ideals (or dual ideals) of a lattice. In the present paper, we shall use this technique to study the set of all coverings of a topological space.

The paper is in four parts. The first contains the topological preliminaries and definitions. In the second part, the concept of a covering ideal is introduced and the correspondence between covering ideals and topological spaces is proved. Part three shows how continuous mappings can be interpreted in terms of homomorphisms of covering ideals. Finally, in part four, two simple applications of the theory are presented.

Notation. Set operations will be denoted by rounded symbols. Thus $\cup, \cap, \subseteq$ will stand for set union, intersection, and inclusion respectively. The symbol $\subset$ is reserved for proper inclusion. If $A$ and $B$ are sets, $A-B$ $=\{a \in A \mid a \in B\}$. The complement (in some fixed set) of a set $A$ is written $A^{c}$. Finally, the empty set is denoted $\varnothing$.

Sharp cornered symbols will be used to denote lattice operations or relations. Thus $\vee, \wedge, \leqq$ denote respectively the lattice join, meet, and order. The zero and unit of a lattice will be represented by $o$ and $u$. In a Boolean algebra, the complement of an element $a$ is written $a^{\prime}$.

A mapping $\phi$ of elements of some set $A$ to the elements of a set $B$ induces a mapping (again denoted by $\phi$ ) of $2^{A}$ to $2^{B}$ as follows: $\phi\left(A_{1}\right)=\left\{\phi a \mid a \in A_{1}\right\}$ for $A_{1} \subseteq A$.

If $S$ is a topological space and $A$ is any subset of $S$, the closure and interior of $A$ are respectively denoted $A^{-}$and $A^{\circ}$. We observe that $(A \cap B)^{-o}$ $=A^{-\circ} \cap B^{-o}$ if $A$ and $B$ are open sets. An open set $A$ which satisfies $A=A^{-\circ}$ is called regular. The collection of all regular open sets of $S$ forms a complete Boolean algebra which will be denoted by $B(S)$. Any space with a neighborhood basis composed of regular open sets is called semi-regular.

\section{0 . Algebraic introduction.}

It is convenient to collect some algebraic definitions which can not be found in [1] or other standard references.

Received by the editors June 17, 1953.

(1) This work was supported by the Office of Naval Research. The author is indebted to Professor R. P. Dilworth for his helpful discussion of some of the results of the paper. 
Definition 0.1. A subset $I$ of a partially ordered set is called a terminal subset if $a \in I$ implies $b \in I$ for all $b \geqq a$.

Definition 0.2 . Let $P$ be a set which is partially ordered by $\leqq$. Define a quasi-ordering of the set of all subsets of $P$ by $A<B$ if, for any $a \in A$, there exists $b \in B$ satisfying $a \leqq b$.

Some elementary properties of < which will be used often are:

(1) if $A \subseteq B$, then $A<B$,

(2) $A<\varnothing$ if and only if $A=\varnothing$,

(3) if $C$ is a terminal subset of $P$ and $A<B$, then $A \cap C<B \cap C$.

Definition 0.3. Let $P$ be a partially ordered set with a zero $o$. A subset $P^{\prime}$ of $P$ is called dense in $P$, if for any $a \neq 0$ in $P, b \neq 0$ exists in $P^{\prime}$ with $b \leqq a$.

Definition 0.4. A semi-lattice (abbreviated s.l. is a partially ordered set with a zero element in which every pair of elements has a g.l.b. A mapping $\pi$ of one semi-lattice into another is a homomorphism if it satisfies $\pi(a \wedge b)$ $=\pi a \wedge \pi b$ and $\pi o=0$.

REMARK 1. In the literature, the existence of a zero is not included in the definition of a s.l.

REMARK 2. It is possible to define a dual ideal of a s.1. just as a dual ideal of a lattice is defined. In speaking of these, the adjective "dual" will often be omitted. Since lattice ideals in the usual sense will never be considered, this can cause no confusion.

Definition 0.5. Let $P$ be a semi-lattice. Define the commutative and associative operation $\wedge$ on the set of all subsets of $P$ by $A \wedge B=\{a \wedge b \mid a \in A$, $b \in B\}$.

(4) If $A, B$ and $C$ are subsets of $P$, then $A<B \wedge C$ if and only if $A<B$ and $A<C$.

Definition 0.6. A semi-lattice $P$ is disjunctive if, whenever $a \$ b, c \in P$ exists satisfying $a \wedge c \neq 0$ and $b \wedge c=o$.

Remark. If $P^{\prime}$ is a dense sub-s.l. of the disjunctive s.l. $P$, then $P^{\prime}$ is also disjunctive. In particular, any dense sub-s.l. of a Boolean algebra is disjunctive.

Proposition 0.7 (Stone-Glivenko-Büchi). Let $P$ be a disjunctive semilattice. Then there exists a complete Boolean algebra $Q$ and an isomorphism $\pi$ of $P$ onto a dense subset of $Q$. Moreover, $\pi$ is unique in the sense that if $Q_{0}$ is a complete Boolean algebra and $\pi_{0}$ is an isomorphism of $P$ onto a dense subset of $Q_{0}$, then $\pi_{0} \pi^{-1}$ can be extended to an isomorphism of $Q$ onto $Q_{0}$.

Proof. See [2].

\section{PART I. $P$-spaces}

In part one we shall define a class of topological spaces-the $P$-spaces. These are the spaces which will be studied in the remainder of the paper.

1. Fundamental definitions. 
Proposition 1.1. Let $P=\{o, a, b, \cdots\}$ be a semi-lattice with a zero o. Let $S=\{X, Y, \cdots\}$ be a (possibly empty) set of proper (not empty and not all of $P$ ) dual ideals of $P$. Then $S$ becomes a $T_{0}$ topological space (whose points are the distinct ideals $X, Y, \cdots)$ ) if the sets of the form

$$
S(a)=\{X \in S \mid a \in X\}
$$

are taken as an open neighborhood basis. Moreover, $S(a) \cap S(b)=S(a \wedge b)$ and $S(o)=\varnothing$. The mapping $a \rightarrow S(a)$ is one-to-one if and only if the following is satisfied whenever $a \$ b$ :

$\left(\mathrm{M}_{a, b}\right) \quad$ there exists $X \in S$ with $a \in X$ and $b \in X$.

Remark. In general $S(a \bigvee b) \neq S(a) \cup S(b)$. (See 2.2 below.)

Proof of 1.1. It will be sufficient to show that $S(a) \cap S(b)=S(a \wedge b)$. If $X \in S(a) \cap S(b)$, then $a \in X$ and $b \in X$. Since $X$ is a dual ideal, $a \wedge b \in X$ and $X \in S(a \wedge b)$. Therefore $S(a) \cap S(b) \subseteq S(a \wedge b)$. On the other hand, if $a \wedge b \in X$, then $a \in X$ and $b \in X$, so $S(a \wedge b) \subseteq S(a) \cap S(b)$.

Definition 1.2. Let $P$ be a semi-lattice. A space $S$ whose points are proper dual ideals of $P$ will be called a $P$-space if the topology of $S$ is obtained by taking all the sets of the form (1) as a neighborhood basis. If, in addition, $S$ satisfies condition $\mathrm{M}_{a, b}$ whenever $a \neq b$, then $S$ is called a representative $P$ space.

A characterization of those $T_{0}$ spaces which are homeomorphic to $P$ spaces is given by 1.1 and its converse:

Proposition 1.3. Let $P$ be a semi-lattice. Let $S^{\prime}$ be a $T_{0}$ space and $P^{\prime}$ a collection of open sets of $S^{\prime}$ such that (i) $P^{\prime}$ is a basis for the open sets of $S^{\prime}$, (ii) $P^{\prime}$ is closed under intersection and contains the empty set, and (iii) there is a homomorphism $\pi$ of $P$ onto $P^{\prime}$. Let $S$ be the $P$-space whose points are the proper dual ideals

$$
X_{x}=\{a \in P \mid x \in \pi a\}, \quad \text { where } x \in S^{\prime} .
$$

Then the mapping $X_{x} \rightarrow x$ is a homeomorphism of $S$ onto $S^{\prime}$, mapping the open set $S(a)=\left\{X_{x} \in S \mid a \in X_{x}\right\}$ onto $\pi a$. Finally, if $\pi$ is an isomorphism, $S$ is a representative $P$-space.

The following simple results will be needed later.

Lemma 1.4. Let $T$ be an arbitrary subset of the P-space $S$. Then the closure of $T$ is $T^{-}=\{X \in S \mid X \subseteq U T\}$ (where $U T=U\{Y \mid Y \in T\}$ ).

Proof. If $X \subseteq U T$, and if $a \in X$, then $a \in Y$ for some $Y \in T$. Thus every neighborhood of $X$ contains a point of $T$ so that $X \in T^{-}$. Conversely, if $X \in T^{-}$, every neighborhood of $X$ contains a point of $T$. Hence if $a \in X$, then $Y$ exists in $T$ so that $a \in Y$; that is, $X \subseteq U T$. 
Lemma 1.5. A P-space $S$ is a $T_{1}$ space if and only if $X \subset Y$ never holds for $X$ and $Y$ in $S$.

Lemma 1.6. A representative $P$-space $S$ is a Hausdorff space if and only if for any $X$ and $Y$ of $S$ with $X \neq Y$, there exists $a \in X$ and $b \in Y$ such that $a \wedge b=0$.

Lemma 1.7. Let $S_{1}$ and $S_{2}$ be $P$-spaces. Define $S_{1} \leqq S_{2}$ if, for any $X_{1} \in S_{1}$, there exists $X_{2} \in S_{2}$ such that $X_{2} \subseteq X_{1}$. Then $\leqq$ is a quasi-ordering on the set of all $P$-spaces and a partial ordering on the set of all $T_{1} P$-spaces.

In parts two and three of this paper, we shall be concerned mostly with $P$-spaces which are compact and $T_{1}$. It is convenient to introduce some notation for the set of all such spaces.

Definition 1.8. Let $P$ be a semi-lattice. Denote by $\Sigma_{P}$ the set of all compact $T_{1} P$-spaces (including the empty space). Two spaces of $\Sigma_{P}$ are equal if and only if they consist of the same dual ideals. The relation $\leqq$ of 1.7 partially orders $\Sigma_{P}$.

2. Semi-regular $P$-spaces. In this section, we shall examine those $P$-spaces for which $P$ is a complete Boolean algebra.

Theorem 2.1. Let $P$ be a complete Boolean algebra and let $S$ be a representative $P$-space. Then the regular open sets of $S$ are precisely those of the form $S(a)$.

Proof. From the assumption that $S$ is a representative $P$-space, it follows that $S(a)^{-}=\{X \in S \mid a \wedge b \neq 0$ all $b \in X\}$. Thus $S(a)^{-c}=\{X \in S \mid a \wedge b=0$ some $b \in X\}=\left\{X \in S \mid a^{\prime} \in X\right\}=S\left(a^{\prime}\right)$. Hence $S(a)^{-o}=S(a)^{-c-c}=S\left(a^{\prime}\right)^{-c}=S\left(\left(a^{\prime}\right)^{\prime}\right)$ $=S(a)$, that is, $S(a)$ is a regular open set.

Thus the mapping $a \rightarrow S(a)$ is a meet isomorphism of the complete Boolean algebra $P$ onto a dense subset of the complete Boolean algebra of regular open sets of $S$. By 0.7 , this mapping must be onto. That is, every regular open set of $S$ is of the form $S(a)$ for some $a \in P$.

CoRollary 2.2. Let $P$ be a complete Boolean algebra. Then any representative $P$-space is a semi-regular $T_{0}$ topological space whose Boolean algebra of regular open sets is isomorphic to $P$. Conversely, if $P$ is isomorphic to the Boolean algebra of regular open sets of the semi-regular $T_{0}$ space $S$, then $S$ is homeomorphic to a representative $P$-space.

Proof. By 2.1, 1.1, and 1.3.

REMARK. When $P$ is a complete Boolean algebra, Definition 1.2 can be simplified. In fact, if $P$ is a disjunctive semi-lattice, then a $P$-space $S$ is representative if and only if condition $\mathbf{M}_{a, o}$ is satisfied for all $a \neq 0$.

ExAmple 2.3. Let $S$ be a (nonempty) semi-regular $T_{1}$ space which satisfies the second countability axiom and has no isolated points. Then it is proved in $[1$, p. 177] that $B(S)$ (the Boolean algebra of regular open sets of $S$ ) is iso- 
morphic to the completion by cuts of the free Boolean algebra with countably many generators.

Lemma 2.4. Let $S$ be a dense subspace of the topological space $S_{0}$. If $A$ is a regular open set of $S_{0}$, then $A \cap S$ is regular open in $S$ and the mapping $A \rightarrow A \cap S$ is an isomorphism of $B\left(S_{0}\right)$ onto $B(S)$.

Proof. Obvious.

CoROllary 2.5. Let $S$ be a space in which the isolated points are dense. Then $B(S)$ is isomorphic to the Boolean algebra of all subsets of the set of isolated points in $S$.

By combining 2.3 and 2.5 , it is possible to characterize $B(S)$ whenever $S$ is a semi-regular $T_{1}$ space which satisfies the second countability axiom.

\section{PART II. Covering IDEALS}

The chief object of part two is to formulate and prove the main theorem of the paper (Theorem 4.5). In the first section, we introduce the concept of a covering ideal and show how $P$-spaces give rise to covering ideals. The precise relation between $P$-spaces and covering ideals is established in the next section. Finally, the covering ideals which correspond to compact Hausdorff spaces are characterized.

Throughout part two, $P$ will denote a fixed semi-lattice. In the notation, explicit indication of dependence on $P$ will be omitted when there is no danger of confusion.

\section{Covering ideals.}

Definition 3.1. A finite, nonempty subset $\alpha$ of $P$ is called a $P$-covering (or simply a covering). The collection of all $P$-coverings will be denoted $\mathcal{L}$ $\left(=\mathcal{L}_{P}\right.$ ). For $\alpha, \beta \in \mathcal{L}$, define $\alpha<\beta$ and $\alpha \wedge \beta$ as in 0.2 and 0.5 .

A nonempty subset $\mathfrak{a}$ of $\mathcal{L}$ is called a $P$-covering ideal (or just a covering ideal) if it has the properties:

(i) $\alpha_{1}, \alpha_{2} \in \mathfrak{a}$ implies $\alpha_{1} \wedge \alpha_{2} \in \mathfrak{a}$,

(ii) $\alpha \in \mathfrak{a}$ and $\beta>\alpha$ implies $\beta \in \mathfrak{a}$.

The set of all $P$-covering ideals will be denoted $\Gamma\left(=\Gamma_{P}\right)$. Let $\Gamma$ be partially ordered by inclusion.

Definition 3.2. Let $S$ be a $P$-space. If $\alpha \in \mathcal{L}$ satisfies $S=\bigcup\{S(a) \mid a \in \alpha\}$, or equivalently, $X \cap \alpha \neq \varnothing$ for all $X \in S$, then $\alpha$ is called a covering of $S$. Denote by $\mathfrak{a}(S)$ the set of all coverings of $S$.

Our first lemma is an obvious consequence of the definitions.

Lemma 3.3. For any $P$-space $S, \mathfrak{a}(S)$, if it is not empty, is a covering ideal. If $S_{1} \leqq S_{2}$ (see 1.7), then $\mathfrak{a}\left(S_{1}\right) \supseteq \mathfrak{a}\left(S_{2}\right)$. If $S$ is the empty space, $\mathfrak{a}(S)=\mathcal{L}$.

Definition 3.4. Let $\mathfrak{a}$ be a covering ideal and let $(a, b)$ be an ordered pair 
of elements of $P$. Then $\mathfrak{a}$ is called a representative covering ideal if the following condition is satisfied whenever $a \ddagger b$ :

$\left(\mathrm{N}_{a, b}\right)$ there exists $\alpha \in \mathfrak{a}$ such that $a, b \in \alpha$ and $\alpha-\{a\} \oplus \mathfrak{a}$.

For convenience, $\mathrm{N}_{a, o}$ can be replaced by the equivalent

$\left(\mathrm{N}_{a}\right)$ there exists $\alpha \in \mathfrak{a}$ such that $\alpha-\{a\} \notin \mathfrak{a}$.

Lemma 3.5. Let $S \in \Sigma_{P}$ (see 1.8), and let $(a, b)$ be an ordered pair of elements of $P$. Then $\mathfrak{a}(S)$ satisfies $\mathrm{N}_{a, b}$ if and only if $S$ satisfies $\mathrm{M}_{a, b}$ of 1.1. In particular $\mathfrak{a}(S)$ satisfies $\mathrm{N}_{a}$ if and only if $a \in X$ for some $X \in S$.

Proof. If $S$ satisfies $\mathrm{M}_{a, b}, X \in S$ exists so that $a \in X, b \in X$. Suppose $Y$ is a point of $S$ different from $X$. Since $S$ is a $T_{1}$ space, there exists $c \in Y$ with $c \notin X$. By compactness, choose $c_{1}, \cdots, c_{n}$ so that $c_{i} \notin X$ for all $i$ and $\alpha$ $=\left\{a, b, c_{1}, \cdots, c_{n}\right\}$ is a covering of $S$. Since $c_{i} \notin X$ and $b \notin X, \alpha-\{a\}$ $=\left\{b, c_{1}, \cdots, c_{n}\right\} \notin \mathfrak{a}(S)$. Hence $\mathfrak{a}(S)$ satisfies $\mathrm{N}_{a, b}$.

Conversely, if $\mathfrak{a}(S)$ satisfies $\mathrm{N}_{a, b}$, then $\alpha \in \mathfrak{a}(S)$ exists with $a, b \in \alpha$ and $\alpha-\{a\} \notin \mathfrak{a}(S)$. This means $(\alpha-\{a\}) \cap X=\varnothing$ for some $X \in S$. In particular $b \notin X$. Since $\alpha \in \mathfrak{a}(S), a \in X$. Thus $S$ satisfies $\mathrm{M}_{a, b}$.

Corollary 3.6. If $S \in \Sigma, \mathfrak{a}(S)$ is a representative covering ideal if and only if $S$ is a representative $P$-space (Definition 1.2).

Now we shall determine the algebraic relation between $\mathfrak{a}(S)$ and the points (dual ideals) of the compact $T_{1} P$-space $S$.

Definition 3.7. Let $\mathfrak{a}$ be a covering ideal. A dual ideal $I$ of $P$ is said to be under $\mathfrak{a}$ if $\alpha \cap I \neq \varnothing$ for all $\alpha \in \mathfrak{a}$.

Lemma 3.8. Let $S \in \Sigma$ and assume $S$ is not empty. Suppose $I$ is a dual ideal of $P$ which is under $a(S)$. Then there is a point $X$ of $S$ with $X \subseteq I$.

Proof. Otherwise, for each $X \in S$, there exists $a \in X$ with $a \notin I$. By compactness, it would be possible to choose $\alpha=\left\{a_{1}, \cdots, a_{n}\right\}$ (nonempty) covering $S$ with $a_{i} \notin I$ for all $i$. Then $\alpha \in \mathfrak{a}(S)$ and $\alpha \cap I=\varnothing$, contrary to the assumption that $I$ is under $\mathfrak{a}(S)$.

Lemma 3.9. Let $S \in \Sigma$. Then every point of $S$ is minimal in the set of dual ideals under $\mathfrak{a}(S)$.

Proof. Clearly, every point of $S$ is under a $(S)$. Let $X \in S$. Suppose $I$ is under $\mathfrak{a}(S)$ and $I \subseteq X$. By 3.8 , there is a point $Y$ in $S$ with $Y \subseteq I \subseteq X$. Since $S$ is a $T_{1}$ space, this implies $X=Y=I$. Thus $X$ is minimal under $\mathfrak{a}(S)$.

COROLlaRy 3.10. The proper dual ideals of $P$ which are minimal under $\mathfrak{a}(S)$ are precisely the points of $S$.

Proof. By 3.9, every point of $S$ is minimal under $\mathfrak{a}(S)$. Conversely, if $S \neq \varnothing$, and if $I$ is minimal under $\mathfrak{a}(S)$, there is a point $X$ of $S$ with $X \subseteq I$. By minimality, this implies $X=I$. If $S$ is empty, $\mathfrak{a}(S)=\mathcal{L}$ and no proper ideal is under $\mathfrak{a}(S)$. 


\section{The fundamental theorem.}

Definition 4.1. For $\mathfrak{a} \in \Gamma$, denote by $S(\mathfrak{a})$ the $P$-space of all proper dual ideals of $P$ which are minimal under a.

Corollary 3.10 shows that for any $S_{0} \in \Sigma, S\left(\mathfrak{a}\left(S_{0}\right)\right)=S_{0}$. The remainder of this section will be devoted to showing that for any $\mathfrak{a}_{0} \in \Gamma, \mathfrak{a}\left(S\left(\mathfrak{a}_{0}\right)\right)=\mathfrak{a}_{0}$. First we will prove an existence theorem for dual ideals of $P$.

Proposition 4.2. Suppose $\mathfrak{a} \in \Gamma$ and $\mathfrak{a} \neq \mathcal{L}$. Let $\gamma$ be a collection of coverings such that (i) if $\beta_{1}, \beta_{2} \in \gamma$, then $\beta_{1} \cup \beta_{2} \in \gamma$, and (ii) a $\gamma \gamma=\varnothing$. Then a proper dual ideal $X$ exists which is minimal under a and satisfies $X \cap \beta=\varnothing$ for all $\beta \in \gamma$.

Proof. By the maximal principle, extend $\gamma$ to a collection of coverings $\gamma_{0}$ which is maximal satisfying (i) and (ii). Since $\{0\} \notin \mathfrak{a}, \gamma_{0} \neq \varnothing$. Define $J=\bigcup\left\{\beta \mid \beta \in \gamma_{0}\right\}$.

If $\alpha=\left\{a_{1}, \cdots, a_{n}\right\} \in \mathfrak{a}$, put $\alpha^{*}=\left\{a_{i} \in \alpha \mid a_{i} \leq b\right.$, all $\left.b \in J\right\}$. Then $\alpha^{*} \neq \varnothing$, since otherwise, for each $a_{i} \in \alpha, b_{i}$ and $\beta_{i}$ exist with $a_{i} \leqq b_{i} \in \beta_{i} \in \gamma_{0}$. This implies $\alpha<\beta_{1} \cup \ldots \cup \beta_{n}$, contrary to $\mathfrak{a} \cap \gamma_{0}=\varnothing$.

Put $X=J^{c}$. Then $X \cap \beta=\varnothing$ for all $\beta \in \gamma$ and $\alpha^{*} \subseteq X$ for all $\alpha \in \mathfrak{a}$. Consequently, $X \cap \alpha \neq \varnothing$ for all $\alpha \in \mathfrak{a}$. It must be shown that $X$ is a dual ideal.

If $a \in X,\{a\} \notin \gamma_{0}$, so by the maximality of $\gamma_{0}, \alpha \in \mathfrak{a}$ and $\beta \in \gamma_{0}$ exist satisfying $\alpha<\beta \cup\{a\}$. This is possible only if $\alpha^{*}<\{a\}$. Conversely, $\alpha^{*}<\{a\}$ obviously implies $a \in X$.

If $b \geqq a$ and $a \in X$, then $\alpha^{*}<\{a\}<\{b\}$ for some $\alpha \in \mathfrak{a}$. Hence $b \in X$. If $a_{1}, a_{2} \in X$ and $\alpha_{1}, \alpha_{2} \in \mathfrak{a}$ satisfy $\alpha_{1}^{*}<\left\{a_{1}\right\}, \alpha_{2}^{*}<\left\{a_{2}\right\}$, then $\left(\alpha_{1} \wedge \alpha_{2}\right)^{*}<\alpha_{1}^{*} \wedge \alpha_{2}^{*}$ $<\left\{a_{1}\right\} \wedge\left\{a_{2}\right\}=\left\{a_{1} \wedge a_{2}\right\}$. Hence $a_{1} \wedge a_{2} \in X$ and $X$ is a proper ideal.

Finally, to show that $X$ is minimal under $\mathfrak{a}$, suppose $I$ is a dual ideal under $\mathfrak{a}$ which is contained in $X$. Let $\gamma_{1}=\{\beta \in \mathcal{L} \mid \beta \cap I=\varnothing\}$. Then $\gamma_{1}$ satisfies (i) and (ii) and $\gamma_{1} \supseteq \gamma_{0}$. Since $\gamma_{0}$ is maximal, $\gamma_{1}=\gamma_{0}$. If $a \in X,\{a\} \notin \gamma_{0}=\gamma_{1}$, so $a \in I$. Thus $X \subseteq I$ and the proof is complete.

Corollary 4.3. Suppose $\mathfrak{a} \in \Gamma$ and $\mathfrak{a} \neq \mathcal{L}$. Let $I$ be a dual ideal which is under a. Then there exists a proper dual ideal $X \subseteq I$ which is minimal under $\mathfrak{a}$.

Proof. Put $\gamma=\{\beta \in \mathcal{L} \mid \beta \cap I=\varnothing\}$. Then $\gamma$ satisfies (i) and (ii) of 4.2. Hence a proper ideal $X$ exists which is minimal under $\mathfrak{a}$ and satisfies $X \cap \beta$ $=\varnothing$ for all $\beta \in \gamma$. If $a \in X$, then $\{a\} \notin \gamma$, so $a \in I$. Therefore $X \subseteq I$.

Lemma 4.4. If $\mathfrak{a}$ is a covering ideal, then $S(\mathfrak{a}) \in \Sigma$.

Proof. If $\mathfrak{a}=\mathcal{L}$, then $S(\mathfrak{a})$ is the empty space. Suppose then that $\mathfrak{a} \neq \mathcal{L}$. It must be shown that $S(\mathfrak{a})$ is a compact $T_{1}$ space. If $X_{1}$ and $X_{2}$ are minimal under $\mathfrak{a}$, then $X_{1} \subset X_{2}$ is impossible; thus, by $1.5, S(\mathfrak{a})$ is a $T_{1}$ space.

To prove that $S(\mathfrak{a})$ is compact, it is sufficient to show that if $Q$ is any subset of $P$ with the property $S(\mathfrak{a})=\cup\{S(a) \mid a \in Q\}$ (where $S(a)=\{X \in S(\mathfrak{a}) \mid a$ $\in X\}$ ), there is a finite subset of $Q$ which is in $a$. Suppose otherwise: $a \cap \gamma=\varnothing$, where $\gamma$ denotes the collection of all nonempty finite subsets of $Q$. Then 
$\gamma \subseteq \mathcal{L}$ and $\gamma$ is closed under finite unions, so by 4.2 there is a proper ideal $X$ which is minimal under $\mathfrak{a}$ and satisfies $X \cap \beta=\varnothing$ for all $\beta \in \gamma$. Thus $X \in S(\mathfrak{a})$, and $a \in X$ for all $a \in Q$. This contradicts $S(\mathfrak{a})=\bigcup\{S(a) \mid a \in Q\}$ and shows that some finite subset of $Q$ covers $S(\mathfrak{a})$. Hence $S(\mathfrak{a})$ is compact.

THEOREM 4.5. The mapping $S_{0} \rightarrow \mathfrak{a}\left(S_{0}\right)$ and $\mathfrak{a}_{0} \rightarrow S\left(\mathfrak{a}_{0}\right)$ defines a Galois connection (see [1]) between $\Sigma$ and $\Gamma$. Moreover, if $S_{0} \in \Sigma$ and $\mathfrak{a}_{0} \in \Gamma$, then $S\left(\mathfrak{a}\left(S_{0}\right)\right.$ ) $=S_{0}$ and $\mathfrak{a}\left(S\left(\mathfrak{a}_{0}\right)\right)=\mathfrak{a}_{0}$.

Proof. The first assertion follows from 3.3, 4.4, and Definitions 3.2 and 4.1. By 3.10, $S\left(\mathfrak{a}\left(S_{0}\right)\right)=S_{0}$. The only thing left to prove is $\mathfrak{a}\left(S\left(\mathfrak{a}_{0}\right)\right) \subseteq \mathfrak{a}_{0}$. Assume $\mathfrak{a}_{0} \neq \mathcal{L}$. If $\alpha \notin \mathfrak{a}_{0}$, then by 4.2 there exists $X$ which is minimal under $\mathfrak{a}_{0}$ and satisfies $\alpha \cap X=\varnothing$. Hence $X \in S\left(\mathfrak{a}_{0}\right)$ and $\alpha \notin \mathfrak{a}\left(S\left(\mathfrak{a}_{0}\right)\right)$. Thus $\mathfrak{a}\left(S\left(\mathfrak{a}_{0}\right)\right)$ $\subseteq \mathfrak{a}_{0}$. If $\mathfrak{a}_{0}=\mathcal{L}$, then $S\left(\mathfrak{a}_{0}\right)=\varnothing$ and $\mathfrak{a}\left(S\left(\mathfrak{a}_{0}\right)\right)=\mathcal{L}=\mathfrak{a}_{0}$.

EXAMPLE 4.6. Let $P$ be a Boolean algebra and define $a=\left\{\alpha \in \mathcal{L}_{P} \mid V_{\alpha}=u\right\}$ (where $\mathrm{V} \alpha$ denotes $\mathrm{V}\{a \mid a \in \alpha\}$ ). Suppose $I$ is a proper dual ideal of $P$ which is under a. Then if $a \in P,\left\{a, a^{\prime}\right\} \in \mathfrak{a}$, so $\left\{a, a^{\prime}\right\} \cap I \neq \varnothing$. That is, either $a \in I$ or $a^{\prime} \in I$. Hence $I$ is maximal. Conversely, if $I$ is a maximal proper dual ideal of $P$, then $I$ is prime. Thus if $\alpha \in \mathfrak{a}, \bigvee \alpha=u \in I$ and therefore $\alpha \cap I$ $\neq \varnothing$. This proves that the ideals which are minimal under $\mathfrak{a}$ are precisely the maximal dual ideals of $P$. Consequently $S(\mathfrak{a})$ is the Boolean space associated with $P$.

5. Normal covering ideals. In this section, we shall formulate a sufficient condition on a covering ideal $\mathfrak{a}$ in order that $S(\mathfrak{a})$ be a Hausdorff space. The results contained in $\mathbf{5 . 2}$ and $\mathbf{5 . 4}$ below follow directly from Theorems VI 4.4 and V 8.9 in [7] and proofs will be omitted.

Definition 5.1. Let $\alpha, \beta \in \mathfrak{a}$. Then $\alpha$ is a star-refinement of $\beta\left(\alpha^{*}<\beta\right)$ if for any $a \in \alpha$, there exists $b \in \beta$ such that if $c \in \alpha$ and $a \wedge c \neq 0$, then $c \leqq b$.

A sequence of coverings $\alpha_{0}, \alpha_{1}, \alpha_{2}, \cdots$ is called normal if $\alpha_{j+1}{ }^{*}<\alpha_{j}$ for all $j$. If $\mathfrak{a}$ is a covering ideal, then $\alpha$ is called normal in $\mathfrak{a}$ if $\alpha$ is the first term of a normal sequence of coverings in $\mathfrak{a}$. A covering ideal is called normal if every $\alpha \in \mathfrak{a}$ is normal in $\mathfrak{a}$.

Proposition 5.2. Let $\mathfrak{a}$ be a representative covering ideal. Then $S(\mathfrak{a})$ is Hausdorff if and only if $\mathfrak{a}$ is normal.

Proposition 5.3. Let $\mathfrak{a}$ be a representative covering ideal. Then $S(\mathfrak{a})$ satisfies the second countability axiom if and only if there is a countable subset $A$ of $P$ such that if $\beta \in \mathfrak{a}$, there exists $\alpha \in \mathfrak{a}$ with $\alpha<\beta$ and $\alpha \subseteq A$.

Proof. The sufficiency of this condition is obvious. The necessity follows from 1.1 and 4.5 by an easy compactness argument.

Proposition 5.4. Let a be a representative covering ideal. Then $S(\mathfrak{a})$ is a compactum if and only if there is a normal sequence of coverings which is coinitial in $\mathfrak{a}$. 


\section{PART III. MAPPINGS}

By Theorem 4.5, the coverings rather than the points can be considered as the primitive elements of a compact $T_{1}$ space. This shift of viewpoint can be useful, as the examples of part four will show. It is natural then to try to relate the topological properties of a space directly to the algebraic properties of its covering ideal. With this aim, we have already considered the concepts of Hausdorff separation and perfect separability. In part three the comparison between topology and algebra will be continued with studies of subspaces, homeomorphisms, and continuous mappings of $P$-spaces.

6. Closed subspaces of a $P$-space.

Definition 6.1. Let $S \in \Sigma$. Denote

$$
I(S)=\bigcup\{X \mid X \in S\}=\left\{a \in P \mid \mathfrak{a}(S) \text { satisfies } N_{a}\right\} .
$$

(See 3.4 and 3.5.)

It is evident that $I(S)$ is a terminal subset of $P$ (see 0.1 ). If $P$ is a disjunctive semi-lattice, $I(S)=P-\{0\}$ if and only if $S$ is a representative $P$-space.

Lemma 6.2. Let $S_{0} \in \Sigma$. Suppose $S$ is a closed subspace of $S_{0} . P u t \mathfrak{a}=\mathfrak{a}\left(S_{0}\right)$. Then $S \in \Sigma$ and

(2) if $a \in I(S)$, there exists $\alpha \in \mathfrak{a}$ satisfying $\alpha-\{a\} \ngtr \beta \cap I(S)$ for all $\beta \in \mathfrak{a}$, (3) $\mathfrak{a}(S)=\{\alpha \in \mathcal{L} \mid \alpha>\beta \cap I(S)$, some $\beta \in \mathfrak{a}\}$.

Proof. Obviously $S$ is a compact $T_{1} P$-space, i.e., $S \in \Sigma$. Suppose $a \in I(S)$, Then $a \in X$ for some $X \in S$. By compactness, $\alpha \in \mathfrak{a}$ exists satisfying $X$ $\cap(\alpha-\{a\})=\varnothing$. But $X$ is under $\mathfrak{a}$, so if $\beta \in \mathfrak{a}, \alpha-\{a\} \ngtr \beta \cap X$. Since $X \subseteq I(S)$, this implies (2).

To prove (3), note that if $\alpha>\beta \cap I(S)$ for some $\beta \in \mathfrak{a}$, and if $X \in S$, then $\alpha \cap X>\beta \cap I(S) \cap X=\beta \cap X \neq \varnothing$. Hence $\alpha \in \mathfrak{a}(S)$. Conversely, suppose $\alpha \in \mathfrak{a}(S)$. Let $Y \in S_{0}-S$. Since $S$ is closed, $a \in P$ exists with $Y \in S_{0}(a) \subseteq S_{0}-S$. Thus $a \notin I(S)$. By compactness, $a_{1}, \cdots, a_{n}$ exist with $a_{j} \notin I(S)$ all $j$ and $\beta$ $=\left\{a_{1}, \cdots, a_{n}\right\} \cup \alpha \in \mathfrak{a}$. Then $\alpha=\beta \cap I(S)$.

Lemma 6.3. Let $S_{0} \in \Sigma$. Suppose $S_{1}$ and $S_{2}$ are two closed subspaces of $S_{0}$. Then $I\left(S_{1}\right) \subseteq I\left(S_{2}\right)$ if and only if $S_{1} \subseteq S_{2}$.

Proof. Suppose $S_{1} \Phi S_{2}$. Let $X \in S_{1}, X \notin S_{2}$. Since $S_{2}$ is closed, $a \in P$ exists satisfying $X \in S_{0}(a) \subseteq S_{0}-S_{2}$. Then $a \in I\left(S_{1}\right), a \in I\left(S_{2}\right)$. Thus $I\left(S_{1}\right) \Phi I\left(S_{2}\right)$. The converse is obvious.

Definition 6.4. Let $\mathfrak{a} \in \Gamma$ and suppose $I$ is any terminal subset of $P$. Then $a \in P$ is called essential in $I$ with respect to $\mathfrak{a}$ if $\alpha \in \mathfrak{a}$ exists such that $\alpha-\{a\} \ngtr \beta \cap I$ for all $\beta \in \mathfrak{a}$. If every $a \in I$ is essential in $I$ with respect to $a$, then $I$ is called an $a$-closed subset of $P$. 
If $S$ is a closed subspace of $S(\mathfrak{a})$, then $I(S)$ is $\mathfrak{a}$-closed by (2). The converse is a corollary of the next lemma.

Lemma 6.5. Let $\mathfrak{a} \in \Gamma$ and suppose $I$ is a terminal subset of $P$. Define

$$
S=S(\mathfrak{a}, I)=\{X \in S(\mathfrak{a}) \mid X \subseteq I\} .
$$

Then $S$ is a closed subspace of $S(\mathfrak{a})$ and $I(S)=\{a \in P \mid a$ is essential in $I$ with respect to $\mathfrak{a}\}$.

Proof. By 1.4, $S$ is closed in $S(\mathfrak{a})$. If $a \in I(S)$, then by (2), $a$ is essential in $I(S)$ with respect to a. But $I(S) \subseteq I$ by (1) and (4), so $a$ is essential in $I$ with respect to $a$.

Suppose $a \notin I(S)$, that is, $a \notin X$ for all $X \in S$. Let $\alpha \in \mathfrak{a}$. Then $(\alpha-\{a\})$ $\cap X=\alpha \cap X \neq \varnothing$ for all $X \in S$. If $X \notin S, X \Phi I$ by (4), so $b \in X$ exists with $b \notin I$. By compactness, $\beta=\left\{b_{1}, \cdots, b_{n}\right\} \cup(\alpha-\{a\})$ exists in $a(S(\mathfrak{a}))=\mathfrak{a}$ with $b_{j} \notin I$ for all $j$. Then $\alpha-\{a\}>\beta \cap I$. This proves that $a$ is not essential in $I$ with respect to $a$.

CoRollary 6.6. If $S$ is a closed subspace of $S(\mathfrak{a})$, then $S(\mathfrak{a}, I(S))=S$. If I is an $\mathfrak{a}$-closed subset of $P, I(S(\mathfrak{a}, I))=I$.

\section{Homeomorphisms.}

LEMмA 7.1. Let $\pi$ be an isomorphism of the semi-lattice $P_{1}$ into the semilattice $P_{2}$. Suppose a is a $P_{1}$-covering ideal. Denote by $[\pi \mathfrak{a}]$ the $P_{2}$-covering ideal $\left\{\alpha \in \mathcal{L}_{P_{2}} \mid \alpha>\pi \alpha_{1}, \alpha_{1} \in \mathfrak{a}\right\}$. Then $\pi^{*}: X \rightarrow \pi^{-1} X$ maps $S_{2}=S([\pi \mathfrak{a}])$ homeomorphically onto $S_{1}=S(\mathfrak{a})$.

Proof. A dual ideal $I$ of $P_{2}$ is under $[\pi \mathfrak{a}]$ if and only if $\pi^{-1} I$ is under $\mathfrak{a}$. In particular, if $X \in S_{2}$, then $\pi^{-1} X$ is a point of $S_{1}$. Thus $\pi^{*}$ maps $S_{2}$ into $S_{1}$. If $Y_{1} \in S_{1}$, then $\left[\pi Y_{1}\right]=\left\{a_{2} \in P_{2} \mid a_{2} \geqq \pi a_{1}, a_{1} \in Y_{1}\right\}$ is a dual ideal of $P_{2}$ which is minimal under $[\pi \mathfrak{a}]$. Since $\pi^{*}\left[\pi Y_{1}\right]=\pi^{-1}\left[\pi Y_{1}\right]=Y_{1}, \pi^{*}$ is onto. Also, if $X \in S_{2}$,

$$
\left[\pi\left(\pi^{*} X\right)\right]=X
$$

(because $X$ is minimal under $[\pi \mathfrak{a}]$ ). Thus $\pi^{*}$ is one-to-one. Finally, if $a \in P_{1}$,

$$
\pi^{*} S_{2}(\pi a)=\left\{\pi^{-1} X \mid X \in S_{2}(\pi a)\right\}=\left\{Y_{1} \in S_{1} \mid a \in Y_{1}\right\}=S_{1}(a) .
$$

By (1), the sets $S_{2}(\pi a)$ form a basis for $S_{2}$. Hence $\pi^{*}$ is a homeomorphism.

Theorem 7.2. Let $P_{1}$ and $P_{2}$ be complete Boolean algebras and suppose $\mathfrak{a}_{1}$ and $\mathfrak{a}_{2}$ are representative $P_{1}$ - and $P_{2}$-covering ideals respectively. If $\pi$ is an isomorphism of $P_{1}$ onto $P_{2}$ such that $\pi \mathfrak{a}_{1}=\mathfrak{a}_{2}$, then $\pi$ induces a homeomorphism $\pi^{*}$ of $S_{2}=S\left(\mathfrak{a}_{2}\right)$ onto $S_{1}=S\left(\mathfrak{a}_{1}\right) ; \pi^{*}$ is uniquely determined by the condition $\pi\left(\pi^{*} X\right)$ $=X$. If $\phi$ is a homeomorphism of $S_{2}$ onto $S_{1}$, then $\phi$ induces an isomorphism $\phi^{+}$ of $P_{1}$ onto $P_{2}$ with $\phi^{+} \mathfrak{a}_{1}=\mathfrak{a}_{2} ; \phi^{+}$is uniquely determined by the condition $\phi^{+}(\phi X)$ $=X$. Finally $\left(\pi^{*}\right)^{+}=\pi$ and $\left(\phi^{+}\right)^{*}=\phi$. 
Proof. The theorem follows easily from 7.1.

Corollary 7.3. Let $P$ be a complete Boolean algebra and let $\mathfrak{a}_{1}$ and $\mathfrak{a}_{2}$ be representative $P$-covering ideals. Then $S\left(\mathfrak{a}_{1}\right)$ is homeomorphic to $S\left(\mathfrak{a}_{2}\right)$ if and only if there is an automorphism $\pi$ of $P$ such that $\pi \mathfrak{a}_{1}=a_{2}$.

CoROLlaRy 7.4. Let $P$ be a complete Boolean algebra and let a be a representative covering ideal. If $\phi$ is a homeomorphism of $S(\mathfrak{a})$ onto itself, then $\phi^{+} \mathfrak{a}=\mathfrak{a}$. Conversely, if $\pi$ is an automorphism of $P$ satisfying $\pi \mathfrak{a}=\mathfrak{a}$, then $\pi^{*}$ is a homeomorphism of $S(\mathfrak{a})$ onto itself.

Proof. If $\pi \mathfrak{a}=\mathfrak{a}$ and $X$ is minimal under $\mathfrak{a}$, then $\pi^{*} X=\pi^{-1} X$ is minimal under $\pi^{-1} \mathfrak{a}=\mathfrak{a}$. Hence $\pi^{*}$ maps $S(\mathfrak{a})$ onto itself.

\section{Continuous mappings.}

Theorem 8.1. Let $P_{1}$ and $P_{2}$ be semi-lattices. Suppose $\mathfrak{a}_{1}$ and $\mathfrak{a}_{2}$ are representative $P_{1}$ - and $P_{2}$-covering ideals respectively. Denote $S_{1}=S\left(\mathfrak{a}_{1}\right)$ and $S_{2}=S\left(\mathfrak{a}_{2}\right)$. Assume that $S_{1}$ is a Hausdorff space. Finally, let $\pi$ be a homomorphism of $P_{1}$ into $P_{2}$ which satisfies

$$
\pi \mathfrak{a}_{1} \subseteq \mathfrak{a}_{2}
$$

Then there is a unique continuous mapping $\pi^{*}$ of $S_{2}$ into $S_{1}$ such that

$$
\pi\left(\pi^{*} X\right) \subseteq X, \quad X \in S_{2}
$$

Moreover,

$$
\left(\pi^{*}\right)^{-1} S_{1}(a) \subseteq S_{2}(\pi a) \subseteq\left(\pi^{*}\right)^{-1}\left(S_{1}(a)^{-}\right)
$$

for all $a \in P_{1}$. Finally, $\pi^{*} S_{2}=S\left(\mathfrak{a}_{1}, I\right)$ (see 6.5), where $I=\left\{a \in P_{1} \mid \pi a \neq 0\right\}$.

Proof. Let $X \in S_{2}$. Then $\pi^{-1} X$ is a proper dual ideal of $P_{1}$ which is under $\mathfrak{a}_{1}$. For if $\alpha \in \mathfrak{a}_{1}, \pi \alpha \in \mathfrak{a}_{2}$, so $\pi \alpha \bigcap X \neq \varnothing$. Thus $\alpha \bigcap \pi^{-1} X \neq \varnothing$. By 4.3, $Y \in S_{1}$ exists satisfying $Y \subseteq \pi^{-1} X$. This $Y$ is unique. Indeed, suppose $Y_{1}, Y_{2} \subseteq \pi^{-1} X$, where $Y_{1}$ and $Y_{2}$ are distinct points of $S_{1}$. By 1.6, $o=a \wedge b$ for some $a \in Y_{1}$ and $b \in Y_{2}$. Hence $o \in \pi^{-1} X-$ a contradiction since $\pi o=o \notin X$. Thus (2) defines a unique mapping $\pi^{*}: S_{2} \rightarrow S_{1}$.

To prove (3), notice that $\left(\pi^{*}\right)^{-1} S_{1}(a) \subseteq S_{2}(\pi a)$ follows directly from (2). If $\pi a \in X$, then $a \in \pi^{-1} X$ so that $a \wedge b \neq 0$ for all $b \in \pi^{*} X$. Consequently, $\pi^{*} X \in S_{1}(a)^{-}$, and therefore $S_{2}(\pi a) \subseteq\left(\pi^{*}\right)^{-1}\left(S_{1}(a)^{-}\right)$.

Now suppose $N$ is any open set of $S_{1}$. By (3) and the normality of $S_{1}$, $\left(\pi^{*}\right)^{-1} N=\bigcup\left\{\left(\pi^{*}\right)^{-1} S_{1}(a) \mid S_{1}(a)-\subseteq N\right\} \subseteq \bigcup\left\{S_{2}(\pi a) \mid S_{1}(a)-\subseteq N\right\} \subseteq \bigcup\left\{\left(\pi^{*}\right)^{-1}\right.$ $\left.\cdot\left(S_{1}(a)^{-}\right) \mid S_{1}(a)^{-} \subseteq N\right\}=\left(\pi^{*}\right)^{-1} N$. Thus $\left(\pi^{*}\right)^{-1} N$ is open and $\pi^{*}$ is continuous.

It remains to show that $\pi^{*} S_{2}=S\left(\mathfrak{a}_{1}, I\right)=\left\{Y_{1} \in S_{1} \mid Y_{1} \subseteq I\right\}$. If $X \in S_{2}$, $\pi^{*} X \subseteq \pi^{-1} X=\left\{a \in P_{1} \mid \pi a \in X\right\} \subseteq\left\{a \in P_{1} \mid \pi a \neq 0\right\}=I$. Thus $\pi^{*} S_{2} \subseteq S\left(a_{1}, I\right)$. If $Y_{1} \notin \pi^{*} S_{2}$, then since $\pi^{*} S_{2}$ is closed and $S_{1}$ is normal, $a \in Y_{1}$ exists satisfying $S_{1}(a)-\cap \pi^{*} S_{2}=\varnothing$. By $(3), \quad S_{2}(\pi a) \subseteq\left(\pi^{*}\right)^{-1}\left(S_{1}(a)^{-}\right)=\left(\pi^{*}\right)^{-1}\left[S_{1}(a)-\cap \pi^{*} S_{2}\right]$ 
$=\varnothing$, so $\pi a=o$. Consequently $Y_{1} \nsubseteq I$, that is, $Y_{1} \oplus S\left(\mathfrak{a}_{1}, I\right)$. Since $Y_{1}$ was arbitrary, $S\left(\mathfrak{a}_{1}, I\right) \subseteq \pi^{*} S_{1}$.

REMARK. If $P_{2}$ is a complete Boolean algebra, (3) can be sharpened to

$$
\left[\left(\pi^{*}\right)^{-1} S_{1}(a)\right]^{-0} \subseteq S_{2}(\pi a) .
$$

In order to prove a converse of 8.1 , it is necessary to assume that $P_{2}$ is a complete Boolean algebra. In compensation, it need not be assumed that $S_{1}$ is a Hausdorff space.

Theorem 8.2. Let $P_{1}$ be a semi-lattice and $P_{2}$ a complete Boolean algebra. Let $S_{1}$ and $S_{2}$ be representative $P_{1^{-}}$and $P_{2}$-spaces respectively. Denote $\mathfrak{a}_{1}=\mathfrak{a}\left(S_{1}\right)$ and $\mathfrak{a}_{2}=\mathfrak{a}\left(S_{2}\right)$. Suppose $\phi$ is a continuous mapping of $S_{2}$ into $S_{1}$. Then the relation

$$
S_{2}\left(\phi^{+} a\right)=\left(\phi^{-1} S_{1}(a)\right)^{-0},
$$$$
a \in P_{1},
$$

defines a unique (semi-lattice) homomorphism $\phi^{+}$of $P_{1}$ into $P_{2}$ such that

$$
\phi^{+} \mathfrak{a}_{1} \subseteq \mathfrak{a}_{2}
$$

and

$$
\phi^{+}(\phi X) \subseteq X,
$$$$
X \in S_{2} .
$$

The kernel of $\phi^{+}$is the complement of $I\left(\phi S_{2}\right)$ (see 6.1).

The proof of 8.2 is straightforward and will be omitted.

Theorems 8.1 and 8.2 are converses when the following conditions are fulfilled: $P_{1}$ is a disjunctive semi-lattice, $P_{2}$ is a complete Boolean algebra, $\mathfrak{a}_{1}$ and $\mathfrak{a}_{2}$ are representative covering ideals, and $S_{1}=S\left(\mathfrak{a}_{1}\right)$ is a Hausdorff space. If these are satisfied, 8.1 and $8.2 \mathrm{imply}\left(\phi^{+}\right)^{*}=\phi$ for any continuous mapping $\phi$ of $S_{2}$ into $S_{1}$. However, in general, not all homomorphisms $\pi$ of $a_{1}$ into $\mathfrak{a}_{2}$ satisfy $\left(\pi^{*}\right)^{+}=\pi$ (see 9.4).

Proposition 8.3. Let $P_{1}, P_{2}$, and $P_{3}$ be semi-lattices. Suppose $\mathfrak{a}_{1}, \mathfrak{a}_{2}$, and $\mathfrak{a}_{3}$ are representative $P_{1^{-}}, P_{2^{-}}$, and $P_{3^{-}}$covering ideals respectively. Assume $S\left(\mathfrak{a}_{1}\right)$ and $S\left(\mathfrak{a}_{2}\right)$ are Hausdorff spaces. Finally, suppose $\pi_{1}: P_{1} \rightarrow P_{2}$ and $\pi_{2}: P_{2} \rightarrow P_{3}$ are homomorphisms which satisfy $\pi_{1} \mathfrak{a}_{1} \subseteq \mathfrak{a}_{2}$ and $\pi_{2} \mathfrak{a}_{2} \subseteq \mathfrak{a}_{3}$. Then $\left(\pi_{2} \pi_{1}\right)^{*}=\pi_{1}^{*} \pi_{2}^{*}$.

This follows directly from 8.1. The analogous equation $\left(\phi_{2} \phi_{1}\right)^{+}=\phi_{1}^{+} \phi_{2}^{+}$is usually false. (Compare (4) and (5).)

\section{Homeomorphisms into a compact Hausdorff space.}

Lemma 9.1. Let $P_{1}$ and $P_{2}$ be semi-lattices. Let $S_{1}=S\left(\mathfrak{a}_{1}\right), S_{2}=S\left(\mathfrak{a}_{2}\right)$ be representative compact Hausdorff $P_{1^{-}}$and $P_{2^{-}}$spaces respectively. Suppose $\pi$ is a homomorphism of $P_{1}$ into $P_{2}$ with $\pi \mathfrak{a}_{1} \subseteq \mathfrak{a}_{2}$, such that $\pi^{*}$ is a one-to-one mapping of $S_{2}$ into $S_{1}$. Then $\pi \mathfrak{a}_{1}$ is coinitial in $\mathfrak{a}_{2}$.

Proof. Let $c \in P_{2}$. Suppose $c \in X \in S_{2}$. Then $\pi^{*} X \in \pi^{*} S_{2}(c)$ and since 
$\pi^{*}$ is a homeomorphism, $\pi^{*} S_{2}(c)$ is an open set of $\pi^{*} S_{2}$ in its relative topology. Hence, because $S_{1}$ is normal, $a \in P_{1}$ exists satisfying $\pi^{*} X \in S_{1}(a) \cap \pi^{*} S_{2}$ $\subseteq S_{1}(a)^{-} \cap \pi^{*} S_{2} \subseteq \pi^{*} S_{2}(c)$. By $8.1(3), S_{2}(\pi a) \subseteq\left(\pi^{*}\right)^{-1}\left(S_{1}(a)^{-}\right)=\left(\pi^{*}\right)^{-1}\left[S_{1}(a)^{-}\right.$ $\left.\cap \pi^{*} S_{2}\right] \subseteq\left(\pi^{*}\right)^{-1}\left[\pi^{*} S_{2}(c)\right]=S_{2}(c)$. Putting $c=0$ in this last equation also shows that if $S_{1}(a)-\cap \pi^{*} S_{2}=\varnothing$, then $\pi a=0$.

Now suppose $\beta \in \mathfrak{a}_{2}$. By the result of the above paragraph and the compactness of $\pi^{*} S_{2}$, it is possible to choose $a_{1}, \cdots, a_{n}$ in $P_{1}$ so that $\pi^{*} S_{2}$ $\subseteq S_{1}\left(a_{1}\right) \cup \ldots \cup S_{1}\left(a_{n}\right)$ and $\left\{\pi a_{1}, \cdots, \pi a_{n}\right\}<\beta$. Since $S_{1}$ is compact Hausdorff, $b_{1}, \cdots, b_{m}$ exist so that $S_{1}\left(b_{j}\right)-\cap \pi^{*} S_{2}=\varnothing$ for all $j$ and $\alpha=\left\{a_{1}, \cdots, a_{n}\right.$, $\left.b_{1}, \cdots, b_{m}\right\} \in \mathfrak{a}_{1}$. Then by the last statement of the first paragraph, $\pi \alpha$ $<\left\{\pi a_{1}, \cdots, \pi a_{n}\right\}<\beta$.

Corollary 9.2. Let $P_{1}$ be a semi-lattice and $P_{2}$ a complete Boolean algebra. If $\phi$ is a one-to-one continuous mapping of the representative $P_{2}$-space $S\left(\mathfrak{a}_{2}\right)$ into the compact Hausdorff representative $P_{1}$-space $S\left(\mathfrak{a}_{1}\right)$, then $\phi^{+} \mathfrak{a}_{1}$ is coinitial in $\mathfrak{a}_{2}$.

Combining 9.1 with the following theorem, we get a precise characterization of those homomorphisms $\pi$ (with $\pi \mathfrak{a}_{1} \subseteq \mathfrak{a}_{2}$ ) for which $\pi^{*}$ is one-to-one.

Theorem 9.3. Let $P_{1}$ and $P_{2}$ be semi-lattices. Let $S_{1}=S\left(\mathfrak{a}_{1}\right), S_{2}=S\left(\mathfrak{a}_{2}\right)$ be representative $P_{1}$ and $P_{2}$-spaces respectively. Assume $S_{1}$ is compact Hausdorff. Suppose $\pi$ is a homomorphism of $P_{1}$ into $P_{2}$ such that $\pi \mathfrak{a}_{1}$ is a coinitial subset of $\mathfrak{a}_{2}$. Then

(i) $\pi$ maps $P_{1}$ onto a dense subset of $P_{2}$,

(ii) $\pi^{*}$ is a one-to-one mapping of $S_{2}$ into $S_{1}$ and

(iii) if $P_{2}$ is a complete Boolean algebra, $\left(\pi^{*}\right)^{+}=\pi$ if and only if $I$ $=\left\{a \in P_{1} \mid \pi a \neq 0\right\}$ is an $\mathfrak{a}_{1}$-closed subset of $P_{1}$ (see 6.4).

Proof. The assumption that $\pi \mathfrak{a}_{1}$ is coinitial in the representative $P_{2}$-covering ideal $\mathfrak{a}_{2}$ obviously implies (i).

Let $X \in S_{2}$. Denote by $[X]$ the dual ideal $\left\{b_{2} \in P_{2} \mid b_{2} \geqq \pi a, a \in \pi^{*} X\right\}$. By 8.1 (2), $[X] \subseteq X$. It will now be shown that $[X]$ is under $a_{2}$ and therefore (since $X$ is minimal under $\mathfrak{a}_{2}$ ) $X=[X]$. Let $\beta \in \mathfrak{a}_{2}$. Then by hypothesis, $\alpha \in \mathfrak{a}_{1}$ exists so that $\pi \alpha<\beta$. Since $\pi^{*} X$ is under $a_{1}, \pi^{*} X \cap \alpha \neq \varnothing$. Hence $\varnothing \neq[X] \cap \pi \alpha$ $<[X] \cap \beta$. Because $\beta$ was arbitrary, $[X]$ is under $\mathfrak{a}_{2}$ and $[X]=X$. Now if $X$ and $Y$ in $S_{2}$ are such that $\pi^{*} X=\pi^{*} Y$, then $[X]=[Y]$. Consequently $X=Y$. This proves that $\pi^{*}$ is one-to-one. By $8.1, \pi^{*} S_{2}=S\left(\mathfrak{a}_{1}, I\right)$ (see 6.5), where $I$ $=\{a \mid \pi a \neq 0\}$.

Suppose $I$ is $\mathfrak{a}_{1}$-closed. By 6.6, $I=I\left(S\left(\mathfrak{a}_{1}, I\right)\right)$, so $\pi b \neq o$ implies $b \in \pi^{*} X$ for some $X \in S_{2}$. Let $a \in P_{1}$. Suppose $b \in P_{1}$ satisfies $o \neq \pi b \leqq \pi a$. Then for some $X \in S_{2}, b \in \pi^{*} X$ and $\pi a \in[X]=X$. Therefore $\left(\pi^{*}\right)^{-1} S_{1}(b) \cap S_{2}(\pi a) \neq \varnothing$. Since $\pi P_{1}$ is dense in the complete Boolean algebra $P_{2}$, it follows that $\left(\left(\pi^{*}\right)^{-1} S_{1}(a)\right)^{-}$ $\supseteq S_{2}(\pi a)$, so by $8.1(4), S_{2}(\pi a)=\left(\left(\pi^{*}\right)^{-1} S_{1}(a)\right)^{-o}=S_{2}\left(\left(\pi^{*}\right)^{+} a\right)$. Thus $\left(\pi^{*}\right)^{+}=\pi$. Conversely, if $\left(\pi^{*}\right)^{+}=\pi, S_{2}(\pi a)=\left(\left(\pi^{*}\right)^{-1} S_{1}(a)\right)^{-o}$ for every $a \in P_{1}$. Thus 
$\pi a \neq 0$ implies $S_{2}(\pi a) \cap\left(\pi^{*}\right)^{-1} S_{1}(a) \neq \varnothing$, so $X \in S_{2}(\pi a)$ exists with $a \in \pi^{*} X$. Therefore, $I \subseteq \cup\left\{\pi^{*} X \mid X \in S_{2}\right\}=I\left(S\left(\mathfrak{a}_{1}, I\right)\right)$. By 6.5 , it follows that $I$ is $\mathfrak{a}_{1^{-}}$ closed. The proof of the theorem is complete.

EXAMPLE 9.4. We shall use 9.3 to construct a homomorphism $\pi$ which does not satisfy $\left(\pi^{*}\right)^{+}=\pi$.

Let $S_{1}$ be the real closed interval $[-1,1]$. Let $P_{1}$ be the Boolean algebra of regular open sets of this interval. According to 1.3 and $4.5, S_{1}$ is homeomorphic to the compact Hausdorff representative $P_{1}$-space $S\left(\mathfrak{a}_{1}\right)$, where $\mathfrak{a}_{1}$ is the set of $P_{1}$-coverings of $S_{1}$. Define $X_{0}=\left\{a \in P_{1} \mid 0 \in S_{1}(a)\right\}$ and let $I$ be a (proper) maximal dual ideal of $P_{1}$ with $X_{0} \subset I$. Since $X=X_{0}$ is the only point of $S\left(\mathfrak{a}_{1}\right)$ satisfying $X \subseteq I$, it follows from 6.6 that $I$ is not $\mathfrak{a}_{1}$-closed.

Let $P_{2}$ be the two-element Boolean algebra $\{o, u\}$. Define $\pi$ to be the unique homomorphism of $P_{1}$ on $P_{2}$ with the kernel $I^{c}$. This $\pi$ maps $\mathfrak{a}_{1}$ onto the representative $P_{2}$-covering ideal $a_{2}=\{\{u\},\{o, u\}\}$. Thus the hypotheses of 9.3 are satisfied. By 9.3 (iii), $\pi$ cannot satisfy $\left(\pi^{*}\right)^{+}=\pi$.

\section{Part IV. Applications}

Two applications of the theory developed in the preceding parts will now be presented. First, we shall study compactification, the process of imbedding a topological space as a dense subset in a compact space. The second application is a method of obtaining a topological space from a ring or a lattice. No special effort will be made to obtain new results. Our only aim is to exhibit typical applications of Theorem 4.5.

10. Compactification.

The basic theorem on which most of the discussion of this section depends is

Theorem 10.1. Let $S$ be a $T_{1} P$-space (not necessarily compact). Let a be a $P$-covering ideal. Then $S$ is a subspace of $S(\mathfrak{a})$ if and only if (i) $\mathfrak{a} \subseteq \mathfrak{a}(S)$ and (ii) for any $X \in S$ and any $a \in X$, there exists $\alpha \in \mathfrak{a}$ such that $(\alpha-\{a\}) \cap X=\varnothing$. If $S$ is a representative and (i) and (ii) are satisfied, then $S$ is dense in $S(a)$.

Proof. Suppose (i) and (ii) are satisfied. Let $X \in S$. Then $X \cap \alpha \neq \varnothing$ for all $\alpha \in \mathfrak{a}(S)$, so by (i), $X$ is under a. If $D$ is a dual ideal of $P$ with $D \subset X$, there exists $a \in X$ with $a \in D$. By (ii), $\alpha \in \mathfrak{a}$ exists satisfying $D \cap(\alpha-\{a\})$ $\subseteq X \cap(\alpha-\{a\})=\varnothing$. But $a \notin D$, so $\alpha \cap D=\varnothing$. It follows that $X$ is minimal under $\mathfrak{a}$ and therefore $X \in S(\mathfrak{a})$. Since $X$ was arbitrary, $S \subseteq S(\mathfrak{a})$.

Conversely, suppose $S$ is a subset of $S(\mathfrak{a})$. Then a covering of $S(\mathfrak{a})$ is also a covering of $S$, so (i) is satisfied. By 4.5, any point of $S(\mathfrak{a})$ (and hence of $S$ ) has the property (ii). The last statement of the theorem is obvious.

Remark. The $P$-space topology of a subset $S$ of a $P$-space $S_{0}$ is precisely the relative topology of $S$ induced by $S_{0}$.

EXAmple 10.2. Let $P$ be the s.l. of open sets of the $T_{1}$ space $S$. Let a be the set of all open coverings of $S$. The conditions of 10.1 are satisfied and it can be 
shown (see Example 11.7) that $S(\mathfrak{a})$ is homeomorphic to the Wallman compactification of $S$.

Example 10.3. Suppose again that $P$ is the s.l. of open sets of the $T_{1}$ space $S$, but now let a be the set of all (finite) normal coverings of $S$. Then (see [7]) $\mathfrak{a}$ satisfies 10.1 (ii) if and only if $S$ is completely regular. In this case, $S(\mathfrak{a})$ is a Hausdorff compactification and, in fact, it is not hard to prove that $S(\mathfrak{a})$ is homeomorphic to the Tychonoff compactification of $S$.

Example 10.4. By taking $P=B(S)$ and $\mathfrak{a}=$ all $P$-coverings of $S$, Theorem 10.1 gives the following result: in order that a space $S$ be homeomorphic to a dense subset of a semi-regular compact $T_{1}$ space, it is necessary and sufficient that $S$ be a semi-regular $T_{1}$ space which satisfies:

(1) if $x \in A \subseteq S$, where $A$ is a regular open set, then regular open sets $B_{1}, \cdots, B_{n}$ exist such that $x \notin B_{j}$ for all $j$, and $S=A \cup B_{1} \cup \cdots \cup B_{n}$.

It is obvious that (1) is satisfied if $S$ is a regular space. However, examples of semi-regular Hausdorff spaces which do not satisfy (1) can be given.

Example 10.5. Suppose $S$ is a locally compact Hausdorff space. Let $P$ be the s.l. of all open sets $A$ such that either $A^{-}$or $A^{c}$ is compact. If $\mathfrak{a}$ is the set of all $P$-coverings of $S$, the conditions of 10.1 are satisfied, so $S(\mathfrak{a})$ is a compactification of $S$. It is evident that $S(\mathfrak{a})$ has the same $P$-covering ideal as $\dot{S}$, the one-point compactification of $S$. Thus, by $4.5, S(\mathfrak{a})$ is homeomorphic to $\dot{S}$.

EXAmPLE 10.6. Theorem 10.1 can be used to prove the following theorem of Hurewicz [3]:

A separable metric space of dimension $\leqq n$ can be imbedded as a dense subset in a compactum of dimension $\leqq n$.

Proof. Let $P$ be the collection of all open sets of $S$. By 1.3, it can be assumed that $S$ is a $P$-space. Choose a countable neighborhood basis $A_{1}, A_{2}, \cdots$. For each $A_{j}$, let $B_{j i}$ be a sequence of open sets such that $\bigcap_{i} B_{j i}$ $=\left(A_{j}\right)^{c}$. Let $\alpha_{1}, \alpha_{2}, \cdots$ be a simple enumeration of the coverings $\left\{A_{j}, B_{j i}\right\}$. Put $\beta_{1}=\alpha_{1}$ and by induction choose $\beta_{k}(k>1)$ so that (i) $\beta_{k}$ is a $P$-covering of $S$, (ii) $\beta_{k}$ is a star refinement of $\beta_{k-1} \wedge \alpha_{k}$, and (iii) $\beta_{k}$ has order $\leqq n$. Let $\mathfrak{a}$ be the covering ideal generated by the normal sequence $\left\{\beta_{k}\right\}$. Then $a$ fulfills (i) and (ii) of 10.1 by the choice of the $\alpha$ 's. Hence $S(\mathfrak{a})$ is a compactum (by 5.4) of dimension $\leqq n$ and $S$ is homeomorphic to a dense subset of $S(\mathfrak{a})$.

Proposition 10.7. Let $S$ be a representative $T_{1} P$-space. Let $\mathfrak{a}$ and $\mathfrak{b}$ be $P$ covering ideals satisfying $\mathfrak{a} \subseteq \mathfrak{b} \subseteq \mathfrak{a}(S)$. If $\mathfrak{a}$ satisfies (ii) of 10.1 , so does $\mathfrak{b}$. Moreover, if $\mathfrak{a}$ is a normal covering ideal and $\pi$ is the identity isomorphism of $P$, then $\pi^{*}$ (see 8.1) is a continuous mapping of $S(\mathfrak{b})$ onto $S(\mathfrak{a})$ which satisfies $\pi^{*} X=X$ for $X \in S$ and $\pi^{*}(S(\mathfrak{b})-S)=S(\mathfrak{a})-S$.

Proof. By 4.5 and 8.1.

11. Rings and lattices. In this section, we shall show how a topological space can be obtained from a ring or distributive lattice. Instead of consider- 
ing rings and lattices separately however, it is convenient to treat them both as special cases of semi-rings. A semi-ring $R$ is an algebra with two binary operations, addition (written + ) which is associative and commutative, and multiplication (indicated by juxtaposition) which is associative and satisfies the distributive laws $f(g+h)=(f g)+(f h)$ and $(g+h) f=(g f)+(h f)$. An element $o \in R$ is called a zero if it satisfies $f+o=f$ and $f o=o f=o$ for all $f \in R$. An element $1 \in R$ is called a unit if it satisfies $f 1=1 f=f$ for all $f \in R$.

Lemma 11.1. Let $R$ be a semi-ring and suppose $\pi$ is a product homomorphism of $R$ into a semi-lattice $P$. Assume that $P$ has a unit $u$. Let $\mathcal{X}$ be a multiplicative system in $R$, that is, a nonempty subset of $R$ which is closed under multiplication. Define

$$
\begin{aligned}
\mathfrak{a}(\mathcal{X})=a(\mathcal{X} ; \boldsymbol{R}, \pi, P) & \\
& =\left\{\alpha \in \mathcal{L}_{P} \mid \alpha>\left\{\pi f_{1}, \cdots, \pi f_{n}\right\}, f_{1}+\cdots+f_{n} \in \mathcal{X}\right\} .
\end{aligned}
$$

Then $\mathfrak{a}(\mathscr{H} C)$ is a P-covering ideal.

Proof. If $f_{1}+\cdots+f_{n} \in \mathcal{X}$ and $g_{1}+\cdots+g_{m} \in \mathcal{X}$, then $f_{1} g_{1}+\cdots+f_{n} g_{m}$ $=\left(f_{1}+\cdots+f_{n}\right)\left(g_{1}+\cdots+g_{m}\right) \in \mathcal{X}$. Hence $\left\{\pi f_{1}, \cdots, \pi f_{n}\right\} \wedge\left\{\pi g_{1}, \cdots\right.$, $\left.\pi g_{m}\right\}=\left\{\pi\left(f_{1} g_{1}\right), \cdots, \pi\left(f_{n} g_{m}\right)\right\} \in \mathfrak{a}(\mathcal{H})$. Consequently, $\mathfrak{a}(\mathcal{X} C)$ is a covering ideal which always contains $\{u\}$. (In particular, $\mathfrak{a}(\mathscr{X})$ is nonempty.)

Lemma 11.2. If $\mathscr{X}_{1}$ and $\mathscr{X}_{2}$ are multiplicative systems in $R$, and if $\mathscr{X}_{1} \subseteq \mathscr{X}_{2}$, then $\mathfrak{a}\left(\mathscr{X}_{1}\right) \subseteq \mathfrak{a}\left(\mathscr{X}_{2}\right)$.

Proof. Obvious.

LeMma 11.3. Let $\mathcal{X}_{1}$ and $\mathscr{X}_{2}$ be multiplicative systems in $\mathbb{R}$ such that for

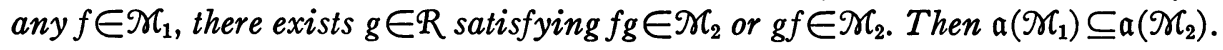

Proof. If $\alpha \in \mathfrak{a}\left(\mathcal{X}_{1}\right)$, then $\alpha>\left\{\pi f_{1}, \cdots, \pi f_{n}\right\}$ where $f_{1}+\cdots+f_{n} \in \mathscr{X}_{1}$. By hypothesis, $g \in R$ exists so that (for instance) $f_{1} g+\cdots+f_{n} g$ $=\left(f_{1}+\cdots+f_{n}\right) g \in \mathcal{X}_{2}$. But $\alpha>\left\{\pi f_{1} \wedge \pi g, \cdots, \pi f_{n} \wedge \pi g\right\}=\left\{\pi\left(f_{1} g\right), \cdots\right.$, $\left.\pi\left(f_{n} g\right)\right\}$, and therefore $\alpha \in \mathfrak{a}\left(\mathcal{X}_{2}\right)$.

CoROLlary 11.4. If $R$ contains a unit 1 , then $\mathfrak{a}(\{1\}) \subseteq \mathfrak{a}(\mathcal{X})$ for any multiplicative system $\mathcal{X}$.

Definition 11.5. Let $R$ be a semi-ring and suppose $\pi$ is a product homomorphism of $R$ into a semi-lattice $P$. Assume that $P$ has a unit. Put

(2) $\mathfrak{a}(\mathbb{R}, \pi, P)=\cap\{\mathfrak{a}(\mathcal{X} ; \mathbb{R}, \pi, P) \mid \mathcal{X}$ is a multiplicative system in $R\}$.

The space $S(\mathfrak{a}(R, \pi, P))$ will be called the $(\pi, P)$-space of $R$.

Lemma 11.6. If $R$ is a semi-ring with a unit 1 and $\pi$ is a product homomorphism of $R$ onto a sub-s.l. of $P$, then $\mathfrak{a}(R, \pi, P)=\mathfrak{a}(\{1\} ; R, \pi, P)$. Moreover, the $(\pi, P)$-space of $R$ is homeomorphic to the $(\pi, \pi R)$-space of $R$. 
Proof. By 11.4 and 7.1.

ExAmple 11.7. Let $P$ be a distributive lattice with a zero and a unit. Then $P$ is also a semi-ring with the operations $\vee$ and $\wedge$ as sum and product respectively. If $\pi_{0}$ is the identity mapping of $P$ onto itself, $\mathfrak{a}=\mathfrak{a}\left(P, \pi_{0}, P\right)$ $=\mathfrak{a}\left(\{u\} ; P, \pi_{0}, P\right)=\left\{\alpha \in \mathcal{L}_{P} \mid \mathrm{V}_{\alpha}=u\right\}$. Suppose $X$ is minimal under $\mathfrak{a}$. If $a \bigvee b \in X$, there exists $\alpha \in \mathfrak{a}$ such that $X \cap(\alpha-\{a \bigvee b\})=\varnothing$. But $(\alpha-\{a \bigvee b\})$ $\cup\{a, b\} \in \mathfrak{a}$, so $a \in X$ or $b \in X$. Thus $X$ is prime. Conversely, if $X$ is prime, $\mathrm{V}_{\alpha}=u \in X$ implies $X \cap \alpha \neq \varnothing$. Consequently, $S(\mathfrak{a})$ is the $P$-space of all minimal prime ideals of $P$. This space is homeomorphic to the Wallman space associated with the dual of $P$ (see [8]).

EXAMPLE 11.8. Let $R$ be the lattice of all real-valued continuous functions on a compact Hausdorff space $S$. Then $R$ is a semi-ring with the lattice operations $(f \vee g)(x)=\max \{f(x), g(x)\}$ and $(f \wedge g)(x)=\min \{f(x), g(x)\}$ as sum and product respectively. Let $\pi$ be the product homomorphism of $R$ into $B(S)$ defined by $f \rightarrow\{x \in S \mid f(x)>0\}^{-0}$. We shall show that the $(\pi, B(S))$-space of $R$ is homeomorphic to $S$.

By 11.2 and 11.3,

$$
\mathfrak{a}(R, \pi, B(S))=\bigcap_{n=1}^{\infty} \mathfrak{a}(\langle n\rangle),
$$

where $\langle n\rangle$ denotes the multiplicative system whose only element is the function on $S$ which is equal everywhere to $n$. If $f_{1} \vee \cdots \vee f_{m} \in\langle n\rangle$, then $S$ $\subseteq \bigcup_{k=1}^{m}\left\{x \in S \mid f_{k}(x)>0\right\}$, so $\left\{\pi f_{1}, \cdots, \pi f_{m}\right\}$ is a covering of $S$. Conversely, by the well known "partition theorem," if $\left\{A_{1}, \cdots, A_{m}\right\}$ is any covering of $S$ by regular open sets, real-valued continuous functions $f_{1}, \cdots, f_{m}$ exist so that $\left\{x \in S \mid f_{k}(x) \neq 0\right\} \subseteq A_{k}$ and $f_{1} \vee \cdots \vee f_{m} \in\langle n\rangle$. Then $\left\{\pi f_{1}, \cdots, \pi f_{m}\right\}$ $<\left\{A_{1}, \cdots, A_{m}\right\}$. Thus $\mathfrak{a}(\langle n\rangle)$ is the set of all $B(S)$-coverings of $S$. By (3), $\mathfrak{a}(R, \pi, B(S))$ is also the set of all $B(S)$-coverings of $S$. It follows from 1.3 and 4.5 that the $(\pi, B(S))$-space of $R$ is homeomorphic to $S$.

REMARK. The homomorphism $\pi$ of 11.8 is uniquely determined by its kernel $\{f \in R \mid f \leqq 0\}$ (see [5]). Thus $\pi$ depends on the zero function. However, it is clear that the result of 11.8 is the same if $\pi$ is replaced by any homomorphism of the form $f \rightarrow\left\{x \in S \mid f(x)>f_{0}(x)\right\}^{-o}$, where $f_{0} \in R$ is fixed. Consequently, 11.8 gives a new proof of the theorem of Kaplansky [4] that a compact Hausdorff space is determined to within homeomorphism by its lattice of real-valued, continuous functions.

EXAMPLE 11.9. Let $R$ be a commutative ring which contains a unit. Let $S$ be the space of maximal proper ideals of $\mathcal{R}$ with the "hull-kernel" topology. Then $S$ is a compact $T_{1}$ space and the collection $P$ of all sets $A_{f}$ $=\{X \in S \mid f \notin X\}$ is a basis for the open sets of $S$. The mapping $\pi: f \rightarrow A_{f}$ is obviously a product homomorphism of $R$ onto the semi-lattice $P$. We shall show that the $(\pi, P)$-space of $\mathbb{R}$ is homeomorphic to $S$.

If $f_{1}+\cdots+f_{n}=1$, and if $X \in S$, then $f_{i} \notin X$ for at least one $i$. For other- 
wise, $1=f_{1}+\cdots+f_{n} \in X$, contrary to the assumption that $X$ is proper. Thus $A_{f_{1}} \cup \ldots \cup A_{f_{n}}=S$. Conversely, if $A_{f_{1}} \cup \ldots \cup A_{f_{n}}=S$, then the ideal generated by $\left\{f_{1}, \cdots, f_{n}\right\}$ is contained in no maximal proper ideal of $R$. Hence $\left\{f_{1}, \cdots, f_{n}\right\}$ generates the unit ideal and $g_{1}, \cdots, g_{n}$ exist such that $g_{1} f_{1}+\cdots+g_{n} f_{n}=1$. This shows that $\mathfrak{a}(\mathcal{R}, \pi, P)=\mathfrak{a}(\{1\} ; \mathcal{R}, \pi, P)$ is the set of all $P$-coverings of $S$. Thus, by 4.5 , the $(\pi, P)$-space of $R$ is homeomorphic to $S$.

ExAmple 11.10 . Let $R$ be a ring of functions on a locally compact Hausdorff space $S$. Assume that the functions of $R$ take their values in an integral domain with a unit. Finally suppose:

(i) for any $f \in R$, the set $\{x \in S \mid f(x) \neq 0\}$ is open,

(ii) if $A$ is open in $S$ and $x \in A$, then $f \in R$ exists so that $f(y)=0$ if $y \in A^{c}$ and $f(y)=1$ for all $y$ in some neighborhood of $x$.

Let $\pi$ be the mapping of $R$ into $B(S)$ defined by $f \rightarrow\{x \in S \mid f(x) \neq 0\}^{-o}$. Because of (i) and (ii), $\pi$ is a product homomorphism which can be defined intrinsically in terms of the ring structure of $R$ (see [5]). Suppose $P$ is a sub-algebra of $B(S)$ which contains $\pi R$. Then it is possible to show that

$$
\mathfrak{a}_{1} \subseteq \mathfrak{a}(R, \pi, P) \subseteq \mathfrak{a}_{0},
$$

where $\mathfrak{a}_{0}$ is the set of all $P$-coverings of $S$ and $\mathfrak{a}_{1}$ is the collection of $P$-coverings which contain at least one regular open set whose complement is compact. Consequently, the $(\pi, P)$-space of $R$ is a compactification of $S$. If $S$ is compact, then by 7.1 , the $(\pi, P)$-space of $R$ is homeomorphic to $S$. Finally, if every element of $R$ has a compact carrier, and if $P$ is the Boolean algebra of those regular open sets $A$ such that either $A^{-}$or $A^{c}$ is compact, then the $(\pi, P)$-space of $R$ is homeomorphic to $\dot{S}$.

\section{REFERENCES}

1. G. Birkhoff, Lattice theory, rev. ed., Amer. Math. Soc. Colloquium Publications, vol. 25, 1949.

2. J. Richard Büchi, Die Boolesche Partialordnung, Portugaliae Mathematica vol. 7 (1948) pp. 118-177.

3. W. Hurewicz, Über Einbettung separabler Räume in gleichdimensionale kompakte Räume, Monatsh. für Mathematik und Physik vol. 37 (1930) pp. 199-208.

4. I. Kaplansky, Lattices of continuous functions, Bull. Amer. Math. Soc. vol. 53 (1947) pp. 617-622.

5. R. Pierce, Homomorphisms of semi-groups, Ann. of Math. vol. 59 (1954) pp. 287-291.

6. M. H. Stone, Applications of the theory of Boolean rings to general topology, Trans. Amer. Math. Soc. vol. 41 (1937) pp. 375-481.

7. J. W. Tukey, Convergence and uniformity in topology, Princeton, 1940.

8. H. Wallman, Lattices and topological spaces, Ann. of Math. vol. 39 (1938) pp. 112-126.

YALE UNIVERSITY,

New Haven, Conn. 